Asian-European Journal of Mathematics

Vol. 7, No. 4 (2014) 1499001 (4 pages)

(c) World Scientific Publishing Company

DOI: 10.1142/S1793557114990010

\title{
Author index (Vol. 7)
}

Adegboyegun, B. J., see Akinwumi

Ahammou, A. \& Iskafi, K., Regular positive solutions to $p$-Laplacian systems on unbounded domain

Ahmadi, M., Moussavi, A. \& Nourozi, V., On skew Hurwitz serieswise Armendariz rings

Akinwumi, T. O. \& Adegboyegun, B. J., On the theory and application of one-step numerical schemes for solving quantum stochastic differential equation (QSDE)

Aldweby, H. \& Darus, M., Partial sum of generalized class of meromorphically univalent functions defined by $q$-analogue of Liu-Srivastava operator

Ansari, A. \& Sheikhani, A. R., New identities for the Wright and the Mittag-Leffler functions using the Laplace transform

Argyros, I. K. \& George, S., On the semilocal convergence of Newton's method for sections on Riemannian manifolds

Argyros, I. K. \& Khattri, S. K., Fixed points for operators with generalized Hölder derivative

Arriola, B. H. \& Canoy, Jr., S. R., Doubly connected domination in the join and Cartesian product of some graphs

Azizi, A., Zekhnini, A. \& Taous, M., On the strongly ambiguous classes of $\mathrm{k} / \mathbb{Q}(i)$ where $\mathbb{k}=\mathbb{Q}\left(\sqrt{2 p_{1} p_{2}}, i\right)$

Balaji, V. K., see Orhan
7 (2014) 1450037

7 (2014) 1450035

7 (2014) 1450036

7 (2014) 1450037

7 (2014) 1450046

7 (2014) 1450038

7 (2014) 1450007

7 (2014) 1450053

7 (2014) 1450054

7 (2014) 1450021

7 (2014) 1450005
Bandaru, R. K., see Rafi

Baskar, S. \& Palaniammal, S., $\mathrm{M} / \mathrm{M}(a, b) /(2,1) \quad$ queueing system with servers repeated and delayed vacation

Butt, S. I., Pečarić, J., Perić, I. \& Praljak, M., Multidimensional reversed Hardy type inequalities for monotone functions

Canoy, Jr., S. R., see Arriola

Chbili, N., A note on the Tutte polynomial and the automorphism group of a graph

Choudhury, B. S. \& Das, P., Coupled coincidence point results for compatible mappings in partially ordered probabilistic metric spaces

Darus, M., see Aldweby

Das, P., see Choudhury

Dhotre, S. B., see Malavadkar

Ehsani, A. \& Movsisyan Yu., A representation of paramedial $n$-ary groupoids

Erfanian, A., see Rezaei

Faroughi, M. H., see Osgooei

Fisher, B., see Lin

George, S., see Argyros

Guo, X. J. \& Shum, K. P., Locally ample semigroup algebras

Hasani, F., see Kheirfam

Hejazi, S. R., Lie group analysis, Hamiltonian equations and conservation laws of Born-Infeld equation

Hong, S. \& Qian, G., On the lcm-analog of binomial coefficient

Ibrahim, Z. B., see Suleiman

Iskafi, K., see Ahammou

Ismail, F., see Suleiman
7 (2014) 1450001

7 (2014) 1450025

7 (2014) 1450032

7 (2014) 1450055

7 (2014) 1450054

7 (2014) 1450009

7 (2014) 1450046

7 (2014) 1450009

7 (2014) 1450060

7 (2014) 1450020

7 (2014) 1450017

7 (2014) 1450041

7 (2014) 1450042

7 (2014) 1450007

7 (2014) 1450067

7 (2014) 1450018

7 (2014) 1450040

7 (2014) 1450056

7 (2014) 1350034

7 (2014) 1450035

7 (2014) 1350034 
Kalorkoti, K., Types of depth and formula size

Kamoun, L. \& Negzaoui, S., An $L^{p}-L^{q}$-version of Morgan's theorem for BesselStruve transform

Karimov, E. T. \& Rakhmatullaeva, N. A., On a nonlocal problem for mixed parabolichyperbolic type equation with nonsmooth line of type changing

Károlyi Gy., Proof of a conjecture of Farhi

Kharat, V. S., see Shewale

Khattri, S. K., see Argyros

Kheirfam, B., A correctorpredictor path-following algorithm for semidefinite optimization

Kheirfam, B. \& Hasani, F., A full-Newton step infeasible interior-point method for linear optimization based on an exponential kernel function

Konieczny, J., Automorphism groups of endomorphism monoids of free $G$-sets

Kovaleva, V. A., Finite groups with generalized $\mathbb{P}$ subnormal second maximal subgroups

Krishna, D. V. \& Ramreddy, T., An upper bound to the nonlinear functional for certain subclasses of analytic functions associated with Hankel determinant

Kurdachenko, L. A., Subbotin, I. Ya. \& Velichko, T. I., On some groups with only two types of subgroups

Lakshmana, A., see Rao

Lal, R. \& Singh, A. K., Weak classification of finite groups

Lanzhe, L., Weighted boundedness for Toeplitz type operator associated to general integral operators

Lehtonen, E., Marichal, J.-L. \& Teheux, B., Associative string functions

Li, Y. K., Yang, L. \& Zhang, H. T., Permanence and uniformly asymptotical stability of almost periodic solutions for a single-species model with feedback control on time scales

7 (2014) 1450004

7 (2014) 1450014

Lin, M., Orankitjaroen, S. \& Fisher, B., The noncommutative neutrix product of $x_{-}^{-s} \ln ^{m} x_{-}$and $x_{+}^{r}$

Lyu, X., Asymptotics in weighted corner spaces

Magesh, N., see Orhan

7 (2014) 1450030

Makhijani, N., Sharma, R. K. \& Srivastava, J. B., The unit group of finite group algebra of a generalized dihedral

7 (2014) 1450044

7 (2014) 1450053

7 (2014) 1450028

7 (2014) 1450018

7 (2014) 1450015

7 (2014) 1450047

7 (2014) 1350042

7 (2014) 1450057

7 (2014) 1450062

7 (2014) 1450058

7 (2014) 1450026

7 (2014) 1450059 group

Malavadkar, P. P., Shikare, M. M. \& Dhotre, S. B., A characterization of $n$-connected splitting matroids

Marichal, J.-L., see Lehtonen

Mishra, A., Sharma, R. K. \& Shukla, W., On the bound of the complexity of the normal basis generated by the trace of the dual element of a Type I optimal normal element

7 (2014) 1450043

Mishra, A. K. \& Soren, M. M., Certain subclasses of multivalent meromorphic functions involving iterations of the Cho-Kwon-Srivastava transform and its combinations

Mojsej, I. \& Tartal' ová, A., On some classes of nonoscillatory solutions of third-order nonlinear differential equations

Moussavi, A., see Ahmadi

Movsisyan, Yu., see Ehsani

Musa, H., see Suleiman

Najati, A., see Osgooei

Nanaji Rao, G., see Rao

Negzaoui, S., see Kamoun

Nourozi, V., see Ahmadi

Orankitjaroen, S., see Lin

Orhan, H., Magesh, N. \& Balaji, V. K., Initial coefficient bounds for certain classes of meromorphic bi-univalent functions

Osgooei, E., Najati, A. \& Faroughi, M. H., g-Riesz dual sequences for g-Bessel sequences
7 (2014) 1450034

7 (2014) 1450042

7 (2014) 1450050

7 (2014) 1450005

7 (2014) 1450060

7 (2014) 1450059

7 (2014) 1450061

7 (2014) 1450039

7 (2014) 1450036

7 (2014) 1450020

7 (2014) 1350034

7 (2014) 1450041

7 (2014) 1450062

7 (2014) 1450014

7 (2014) 1450036

7 (2014) 1450042

7 (2014) 1450005

7 (2014) 1450041 
Palaniammal, S., see Baskar

Pečarić, J., see Butt

Perić, I., see Butt

Praljak, M., see Butt

Qian, G., see Hong

Qiu, J., Gröbner-Shirshov bases for commutative algebras with multiple operators and free commutative RotaBaxter algebras

Rafi, N., Bandaru, R. K. \& Rao, G. C., Dominator and closure ideals in almost distributive lattices

Rakhmatullaeva, N. A., see Karimov

Ramreddy, T., see Krishna

Rao, G. C., see Rafi

Rao, G. C., Nanaji Rao, G. \& Lakshmana, A., Dense elements characterization of quasi-complemented Almost Distributive Lattices

Rao, G. C. \& Sundari Katakam, S. B. T., Stone like duality in almost distributive lattices

Rao, G. C. \& Undurthi, V., Complete almost distributive lattices

Rathee, S., see Ritika

Rezaei, R. \& Erfanian, A., A note on the relative commutativity degree of finite groups

Ritika \& Rathee, S., Thianwan's iteration procedure for nonexpansive mappings in $\mathrm{CAT}(0)$ spaces

Sahai, V. \& Verma, A., $n$ thOrder $q$-derivatives of multivariable $q$-hypergeometric series with respect to parameters

Sambasiva Rao, M., Quasicomplemented $C$-algebras

Santhi Sundar Raj, Ch., Venkateswarlu, B. \& Vasu Babu, R., The Stonity of a pseudo-complemented ADL

Selvaraj, C., Udhayakumar, R. \& Umamaheswaran, A., Gorenstein $n$-flat modules and their covers

Senu, N., see Suleiman

Sethi, A., see Tyagi

Sharma, R. K., see Makhijani
7 (2014) 1450032

7 (2014) 1450055

7 (2014) 1450055

7 (2014) 1450055

7 (2014) 1450056

7 (2014) 1450033

7 (2014) 1450025

7 (2014) 1450030

7 (2014) 1350042

7 (2014) 1450025

7 (2014) 1450062

7 (2014) 1450049

7 (2014) 1450052

7 (2014) 1450003

7 (2014) 1450017

7 (2014) 1450003

7 (2014) 1450019

7 (2014) 1350048

7 (2014) 1450045

7 (2014) 1450051

7 (2014) 1350034

7 (2014) 1350045

7 (2014) 1450034
Sharma, R. K., see Mishra

Sheikhani, A. R., see Ansari

7 (2014) 1450043

7 (2014) 1450038

Shewale, R. S. \& Kharat, V. S., On modular pairs in posets

Shikare, M. M., see Malavadkar

Shukla, W., see Mishra

Shum, K. P., see Guo

Singh, A. K., see Lal

Singh, U. \& Srivastava, S. K., Trigonometric approximation of functions belonging to certain Lipschitz classes by $C^{1} \cdot T$ operator

7 (2014) 1450064

7 (2014) 1450061

Soren, M. M., see Mishra

Soren, M. M., Sandwich results for analytic functions involving with iterations of the Owa-Srivastava operator and its combination

Srivastava, J. B., see Makhijani

Srivastava, S. K., see Singh

Subbotin, I. Ya., see Kurdachenko

7 (2014) 1450044

7 (2014) 1450060

7 (2014) 1450043

7 (2014) 1450067

7 (2014) 1450058

Suleiman, M. B., Musa, H., Ismail, F., Senu, N. \& Ibrahim, Z. B., A new superclass of block backward differentiation formula for stiff ordinary differential equations

Sundari Katakam, S. B. T., see Rao

Taous, M., see Azizi

Tarta ová, A., see Mojsej

Teheux, B., see Lehtonen

Tushev, A. V., On certain methods of studying ideals in group rings of abelian groups of finite rank

7 (2014) 1450065

Tyagi, V. \& Sethi, A., $b_{i}$-Byte correcting perfect codes

7 (2014) 1350045

7 (2014) 1450051

Udhayakumar, R., see Selvara

Umamaheswaran, A., see Selvaraj

7 (2014) 1450051

Undurthi, V., see Rao

7 (2014) 1450052

Vasu Babu, R., see Santhi Sundar Raj, Ch.

Vasu Babu, R., see Venkateswarlu

7 (2014) 1450045

7 (2014) 1450066

Velichko, T. I., see Kurdachenko

7 (2014) 1450057

Venkateswarlu, B., see Santhi Sundar Raj, Ch. 
Venkateswarlu, B. \& Vasu Babu, R., Associate elements in ADLs

Verma, A., see Sahai

Wolf, E., Bellwethers of composition operators for typicalgrowth spaces

Yang, L., see Li

7 (2014) 1450066
7 (2014) 1450019
7 (2014) 1450048
7 (2014) 1450004

Yordzhev, K., On the cardinality of a factor set in the symmetric group

7 (2014) 1450027

Zekhnini, A., see Azizi

7 (2014) 1450021

Zhang, H. T., see Li

7 (2014) 1450004

Zhuchok, Y. V., Representations of ordered dimonoids by binary relations

7 (2014) 1450006 